\title{
Spectral reflectance recovery from a white-balanced RGB image based on the algorithm of compressive sensing
}

\author{
${ }^{1}$ Zhang Leihong, ${ }^{1}$ Liang Dong, ${ }^{1}$ Li Bei, ${ }^{1}$ Kang Yi, ${ }^{2}$ Zhang Dawei and \\ ${ }^{3}$ Ma Xiuhua \\ ${ }^{1}$ College of Communication and Art Design, University of Shanghai for Science and \\ Technology, Shanghai 200093, China \\ ${ }^{2}$ School of Optical, Electrical and Computer Engineering, University of Shanghai \\ for Science and Technology, Shanghai 200093, China \\ ${ }^{3}$ Shanghai Institute of Optics and Fine Mechanics, CAS, Shanghai 201800, China
}

Received: 01.06.2016

\begin{abstract}
In this work we study the methods for reconstructing spectral reflectances of images. To reproduce the spectral reflectance more accurately and the colours more realistically, we obtain a so-called average transformation matrix, using a series of different modulated light sources to illuminate a standard whiteboard. For eliminating the influence of those light sources on RGB images, we first use a white-balance algorithm to standardize the scene information, thus obtaining a uniform RGB image with different sources. Then we map the RGB image onto the spectral reflectance basing on a compressive sensing algorithm and the principal component analysis and, finally, reconstruct the spectral reflectance of the testing sample. We compare the reconstruction accuracies of our compressive sensing algorithm based on white-balance calibration with the results derived using a pseudo-inverse method and a traditional compressive sensing algorithm. Our simulation results show that, under the same conditions of reconstruction, the residual errors and the colour difference resulting from our improved algorithm are less than those produced by the other two algorithms. In other words, the reconstruction algorithm suggested by us outperforms the other methods and can provide better performance of image colour reproduction.
\end{abstract}

Keywords: standard whiteboard, white-balance algorithm, compressive sensing, iterative threshold, spectral reflectance reconstruction

PACS: $42.66 . \mathrm{Ne}$

UDC: 535.62

\section{Introduction}

At present colour reproduction relies mainly on colour space description methods RGB, XYZ or CIELAB, all of which involve some residual inaccuracies of colour reproduction. It is known that the most comprehensive and accurate description of the colour is given by spectral reflectance $[1,2]$. Nonetheless, the reconstructed colour differences based on the traditional methods in this field (see, e.g., Refs. [3-5]) are not sufficiently small while the calculation times remain long enough. The authors of Ref. [6] have used a compressive sensing algorithm to reconstruct the spectral reflectance. It reduces the reconstruction errors and the colour difference, and shortens the calculation times if compared with the pseudo-inverse and Wiener algorithms. In Ref. [7], the spectral reflectance reconstruction based on the algorithm of adaptive compressive sensing has been suggested, which takes into account the representation and the relevance of sample selection and so improves clearly the reconstruction accuracy. However, both of the methods $[6,7]$ require 
huge training samples to obtain a so-called 'average transformation matrix' for reconstructing spectral reflectance with specific light sources. As a result, the reconstruction precision is notably affected by a set of training samples and a light source chosen.

In this work we propose a new method for obtaining the average transformation matrix. Our light source is modulated, and a standard whiteboard is illuminated one by one using the modulated source with a specific relative spectral power distribution. An RGB camera is used to collect the information on the spectral energy, which enables obtaining the camera response. The average transformation matrix is obtained using the advantage that the reflectivity of the standard whiteboard is one hundred percent. In order to avoid the influence of different light sources on the RGB image and obtain a consistent average transformation matrix, we process the image according to the white-balance algorithm. In this manner a uniform average transformation matrix can be obtained. The mapping function between the RGB image and the spectral reflectance is ascertained with the compressive sensing algorithm, using an iterative threshold based on the principal component analysis. In brief, our approach intends to adopt the compressive sensing algorithm [8-12] based on the white-balance correction to reconstruction if spectral reflectances of images. It improves both the spectral reflectance reconstruction efficiency and the precision.

\section{Spectral reflectance reconstruction based on pseudo-inverse algorithm}

When the spectral imaging system obtains a channel value of an image, the output image channel value is affected by the relative spectral energy distribution of a standard lighting $l(\lambda)$, the spectral reflectance $r(\lambda)$ of that image, the sensitivity of a camera $s(\lambda)$, and the spectral transmittance of a colour filter $\tau_{k}(\lambda)$. The process may be described as follows:

$$
u_{k}=\int_{\lambda_{\min }}^{\lambda_{\max }} s(\lambda) l(\lambda) r(\lambda) \tau_{k}(\lambda) d \lambda,
$$

where $k$ is the number of channel in a spectral imaging system and $u_{k}$ the output image signal in the channel $k$. Eq. (1) can be expressed using a discrete matrix,

$$
u_{k}=M^{T} r
$$

where $M^{T}$ represents the spectral response matrix. The parameter $Q=M\left(M^{T} M\right)^{-1}$ can be obtained by the least square method as $\min \|Q u-r\|_{2}^{2}$. Then the spectral reflectance can be obtained $[6,7]$ :

$$
r=M\left(M^{T} M\right)^{-1} u_{k}
$$

\section{Description of our algorithm}

\subsection{Building average transformation matrix $M$ with the standard whiteboard}

We define an average transformation matrix as a matrix that relates the parameters of the camera response $(D)$ and the light source $(L)$. First, a series of lighting source $L=\left(L_{1}, L_{2}, \ldots L_{M}\right)$ with a specific, known in advance relative spectral power distribution is constructed to illuminate a standard whiteboard. Then the matrix $L \times R$ is obtained by combining the spectral reflectance of the whiteboard with the light source. Eq. (2) may be represented using the following discrete matrix: 


$$
u=D \times\left(\begin{array}{l}
L_{11}, L_{12}, \ldots L_{1 N} \\
L_{21}, L_{22}, \ldots L_{2 N} \\
\ldots \ldots \ldots . . \\
L_{M 1}, L_{M 2}, \ldots L_{M N}
\end{array}\right) \times R=D \times L \times R .
$$

Here $D$ is the camera response parameter, which involves the camera sensitivity $s(\lambda)$ and the spectral transmittance of a filter $\tau_{k}(\lambda)$, and $R$ denotes the spectral reflectance of the standard whiteboard. Notice that the channel response $u$ of the RGB camera is a known quantity, which is obtained using the camera to receive the information on the spectral energy distribution. We remind at this point that the standard whiteboard transmittance is equal to $100 \%$. When the modulated light source illuminates the whiteboard, its spectral reflectance amounts to unity, as can be seen from the formula

$$
u=D \times L \times 1=D \times L,
$$

Then the camera response parameter $D$ can be obtained with the pseudo-inverse method as

$$
D=\operatorname{Pin} v(L) \times u \text {. }
$$

Finally, we obtain the transformation matrix as $M=L \times D$, which is based on the parameter $L$ of the structured modulated light source and the camera response parameter $D$.

Hence, the average transformation matrix $M$ can be obtained from the spectral reflectance even though the channel response value of the training sample remains unknown. Then the mapping of the channel response onto the spectral reflectance can be found according to the average transformation matrix thus obtained. The relevant procedures are based on the algorithm of compressive sensing and the principal component analysis. The performance of the matrix $M$ obtained with this method should be certainly high because the standard whiteboard transmits oneto-one the information on the spectral reflectance, thus containing indirectly the information on the image lighting and the camera parameters. On the other hand, the $M$ matrix obtained using the training samples is affected by the parameters of those samples. This cannot ensure that it contains the complete information on all the testing samples.

\subsection{Mapping of $u$ onto $r$}

According to the property of smoothness of the spectral reflectance, $r$ is combined linearly with the feature vector $J$. One can express the linear model as

$$
r=B a=\left[b_{1}, b_{2}, \cdots, b_{J}\right]\left[a_{1}, a_{2}, \cdots, a_{J}\right]^{T},
$$

where $r$ implies the spectral reflectance vector of $N \times 1$ dimensions, $B$ the basis function vector of $N \times J$ dimensions, and $a$ the $J \times 1$-dimension coefficient of the basis function vector. According to the above analysis, we express Eq. (2) in the form

$$
u_{k}=M^{T} r=M^{T} B a,
$$

where $u_{k}$ represents a $K$-dimension vector. Since we have $K<N$, Eq. (2) does not have a definite solution. The $r$ parameter may be expressed as $r=B a$, so that $r$ represents a $J$-dimension vector. Eq. (2) will have a definite solution whenever we have $J<K$. The quantity $B=\left(b_{1}, b_{2}, b_{3}\right)$ can be obtained using the principal component analysis. Since the coefficient $a$ can be found with the iterative threshold algorithm, the mapping from the RGB image $u$ onto the spectral reflectance $r$ 
is given by the formula $[6,7]$

$$
\hat{r}=B\left\{\arg \min _{a}\left(\left\|u-M^{T} B a\right\|_{2}^{2}+\mu\|a\|_{1}\right)\right\} .
$$

In Eq. (9), $\hat{r}$ represents the reconstructed spectral reflectance, $u$ the channel response of the testing sample, and $M$ is defined as $M=L \square D$. Notice that the parameter $\mu$ is introduced to balance the proportions of the two parts of the objective function. Finally, $f$ is used to represent the mapping function from $u$ to $r$.

\subsection{Reconstruction stage}

Let us consider two different spectral reflectances $r_{1}(\lambda)$ and $r_{2}(\lambda)$ resulted from two different kinds of the light source, $L_{1}(\lambda)$ and $L_{2}(\lambda)$. For a given 'observer' function $C_{c}(\lambda)$, these two spectral reflectances share the same RGB values. This 'metamerism problem' is described as

$$
\int L_{1}(\lambda) r_{1}(\lambda) C_{c}(\lambda) d \lambda=\int L_{2}(\lambda) r_{2}(\lambda) C_{c}(\lambda) d \lambda .
$$

When the illumination information is unknown, it is difficult to determine whether the reflectance is $r_{1}(\lambda)$ or $r_{2}(\lambda)$. This is why a single mapping of the illumination information onto the spectral reflectance cannot solve the problem. One straightforward solution is to build the mappings for each illumination. However, this requires a huge effort to calibrate each mapping. When the illumination is different, the RGB image seen by the human eye is different, so the mapping from the RGB image to the spectral reflectance also changes. To provide a uniform mapping, a white-balancing algorithm should be put forward. After obtaining the RGB image, this algorithm must be used to obtain the uniform white-balance image under different light source conditions. This is equivalent to standardizing different light sources. Then for a variety of lighting conditions, the obtained RGB image becomes the same through the white-balance procedure. We represent the step of adding white balancing as

$$
\hat{u}_{k}(\lambda)=\operatorname{diag}\left(\frac{1}{t_{r}}, \frac{1}{t_{g}}, \frac{1}{t_{b}}\right) u_{k}(\lambda) .
$$

Here $t=\left[t_{r}, t_{g}, t_{b}\right]$ is the white balancing vector associated with the white-balancing algorithm chosen. To perform the white-balancing step, we use the shades-of-grey method which is for simplicity based on the Minkowsky norm of the order of $5[13,14]$.

To reconstruct accurately the spectral reflectance of the testing sample image, the image must be first processed using the white balancing. Then the RGB value of the image obtained will always remain the same under the conditions of different light sources. The RGB image is usually obtained for the case of standard light source, as shown in Fig. 1. We define the mapping $f$ as the relationship between the RGB image and the spectral reflectance $r$. Provided that the function $f$ of mapping the RGB image onto the spectral reflectance and the white-balancing image $\hat{u}$ are known, the reconstructed spectral reflectance $\hat{r}(x)$ of the testing sample is given by

$$
\hat{r}(x)=f(\hat{u}(x)) .
$$

The approach suggested by us will be referred to as a 'compressive sensing algorithm based on the white-balance calibration'. 


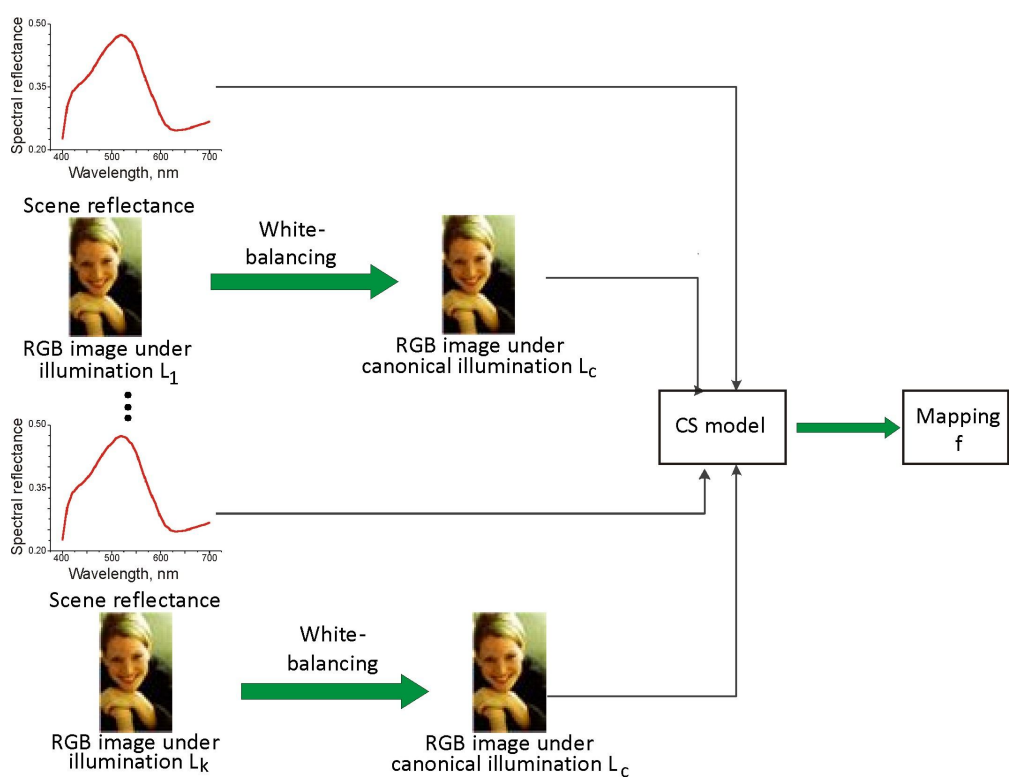

Fig. 1. Mapping relationship between the RGB image and the spectral reflectance.

\section{Experimental results and discussion}

We first construct a series of modulated light sources with specific relative spectral power distributions to illuminate the standard whiteboard. The camera is used to receive the information on the spectral energy distribution. The absolute spectral reflectance ratio for the whiteboard is equal to unity, and the spectral selectivity is small, i.e. we have the same high reflection ratio for each wavelength. Because the transmission rate of the whiteboard is always equal to $100 \%$, the average transformation matrix $M$ can be described as $M=L \times D$. The traditional RGB camera is used to shoot experimental samples of multi-channel spectral images. Six-channel digital response of the testing samples is obtained by adding three different colour filters into the filter gear.

The imaging process is illustrated in Fig. 2. To establish a single uniform mapping from the RGB image to the spectral reflectance, the RGB image first needs to be white-balance corrected. Hence, the white-balance algorithm regulates indirectly the lighting information in the image to obtain uniform RGB images with different light sources. To describe the influence of different algorithms on reconstruction of the spectral reflectance, a simulation experiment has been carried out using the MATLAB software. The image provided by Finland Eastern University (see Ref. [15]) was chosen as a testing sample to reconstruct the spectral reflectance based on our algorithm. The resolution of the original image is $152 \times 91$ and the spectral reflectance range is 400 $700 \mathrm{~nm}$, with intervals of $10 \mathrm{~nm}$, so that the spectral reflectance is collected in $N=31$ dimensions.

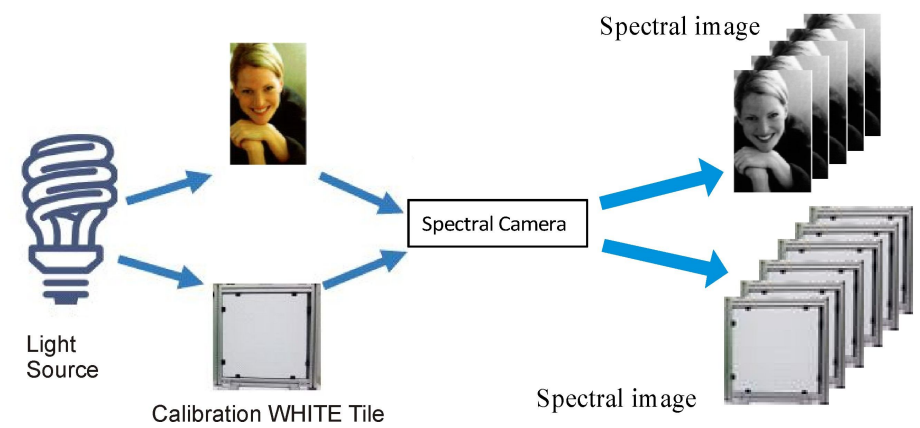

Fig. 2. Illustration of our spectral imaging system. 
The standard colorimetric observer spectral tristimulus values CIE1931 are obtained from the spectral reflectances of multispectral images multiplied by the spectral energy distribution matrix of the standard illuminant D50 and by the standard colorimetric observer spectral matching function CIE1931:

$$
\begin{aligned}
& X=\int_{\lambda_{\min }}^{\lambda_{\max }} \bar{X}(\lambda) l(\lambda) r(\lambda) d \lambda, \\
& Y=\int_{\lambda_{\min }}^{\lambda_{\max }} \bar{Y}(\lambda) l(\lambda) r(\lambda) d \lambda, \quad \\
& Z=\int_{\lambda_{\min }}^{\lambda_{\max }} \bar{Z}(\lambda) l(\lambda) r(\lambda) d \lambda
\end{aligned}
$$

Then through the known colour space conversion matrix, the CIE1931 standard colorimetric observer spectral tristimulus value is transformed into the RGB colour space value. Finally, the RGB image of the testing sample is obtained (see Fig. 3).
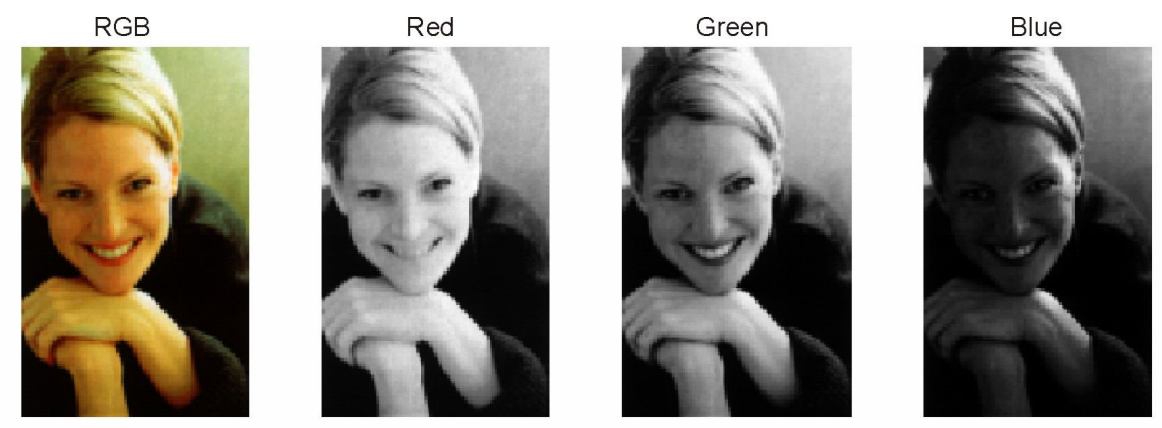

Fig. 3. Multi-spectral images in different channels.

We have used the root-mean-square error to evaluate the precision of spectral reconstruction. Following from the original and reconstructed spectral reflectances $r_{i}$ and $\hat{r}_{i}$ of each pixel, the root-mean-square error can be calculated as

$$
R M S E=\sqrt{\frac{1}{n} \sum_{i=1}^{n}\left(r_{i}-\hat{r}_{i}\right)} .
$$

The chromatic aberration $\Delta E_{a b}$ of the reconstructed spectral reflectance has been evaluated using the CIE1976 chromatic aberration formula. It is based upon the 'coordinates' $\left(L_{1}, a_{1}, b_{1}\right)$ and $\left(L_{2}, a_{2}, b_{2}\right)$ of the original and reconstructed test samples:

$$
\Delta E_{a b}=\left[\left(L_{2}-L_{1}\right)^{2}+\left(a_{2}-a_{1}\right)^{2}+\left(b_{2}-b_{1}\right)^{2}\right]^{1 / 2} .
$$

Here the $L$ component represents a so-called 'lightness', and the $a$ and $b$ components correspond to the changes in the chromatic values from green to red and from blue to yellow, respectively. Finally, we have used the spectral matching precision $G F C$ when evaluating the quality degree of fitting corresponding to the spectral reflectance reconstruction: 


$$
G F C=\frac{\left|\sum_{\lambda} r_{i} \hat{r}_{i}\right|}{\sqrt{\left|\sum_{\lambda} r_{i}^{2}\right|} \sqrt{\left|\sum_{\lambda} \hat{r}_{i}^{2}\right|}} .
$$

\subsection{Spectral reflectance reconstruction based on our method}

Let us denote the spectral reflectance reconstruction process based on the traditional compressive sensing algorithm as CS. It includes the following steps:

(1) The Munsell card is selected as a training sample. Using a camera for obtaining the channel response value of the training sample and a spectrophotometer for measuring the spectral reflectance, one can obtain the average transformation matrix $M$.

(2) The basis function vector $B=\left(b_{1}, b_{2}, b_{3}\right)$ of the spectral reflectance of the training sample is obtained using the principal component analysis, and the first three principal components serve as the components of the basis function vector for the reconstructed spectral reflectance.

(3) Based on the obtained channel response values $u_{k}$ of the testing sample and the first three principal components of the training sample, the sparse coefficient $\hat{a}$ is obtained with the iterative threshold algorithm. Then the spectral reflectance of the testing sample is reconstructed with Eq. (8).

Now we summarize the alternative spectral reflectance reconstruction process based on our algorithm of compressive sensing algorithm with the white-balance correction. The latter method will be referred to as WCS. It includes the following:

(1) The standard whiteboard is placed in front of the camera, and the structured modulated light source (with $L$ known in advance) illuminates the whiteboard. Since the standard whiteboard has the unit reflectance, first the average transformation matrix $M=L \times D$ is obtained.

(2) The compressive sensing algorithm is used to find the mapping function between the RGB image and the spectral reflectance. First, the basis function vector is obtained as the spectral reflectance of the reconstructed testing samples, using the method of principal component analysis. Then the sparse coefficient $\hat{a}$ is obtained, using the compressive sensing algorithm. Finally, the mapping relationship between the RGB image and the spectral reflectance is found as given by Eq. (9).

(3) The RGB images are different due to different light sources. To establish uniform mapping, one has to process the RGB image using the white balance. Then the uniform RGB images are obtained with different sources. Finally, the spectral reflectance of the testing sample is reconstructed with Eq. (12).

Taking one pixel in the image of woman face as an example, we compare reconstructions of the spectral reflectance using the traditional algorithm CS and our algorithm WCS. In particular, the reconstruction error, the colour difference and the spectral matching precision peculiar for the two algorithms are compared, as shown in Table 1. From Table 1 one can draw the following conclusions:

(i) The WCS algorithm can accurately reconstruct the spectral reflectance of the image. The reconstruction error and the colour difference based on this algorithm are smaller than those of the other two algorithms. The GFC parameter associated with the WCS algorithm is larger than that of the CS algorithm; 
(ii) When compared with the CS algorithm which does not take into account the effect of light source, our WCS algorithm standardizes indirectly the lighting of images, so that the reconstruction performance becomes improved.

Table 1. Accuracies of spectral reflectance reconstructions using different algorithms: Pinv, CS and WCS are abbreviations of the pseudo-inverse algorithm, the traditional compressive sensing algorithm and the compressive sensing algorithm based on the white-balance correction, respectively.

\begin{tabular}{|c|c|c|c|}
\hline Method & $R M S E$ & $\Delta E$ & $G F C$ \\
\hline Pinv & 0.013 & 0.75 & 0.9542 \\
\hline CS & 0.0079 & 0.0380 & 0.9677 \\
\hline WCS & 0.000686 & 0.0226 & 0.9705 \\
\hline
\end{tabular}

In Fig. 4 we compare the original spectral reflectance with the corresponding fitting results derived with the methods CS and WCS. A number of conclusions arise as a consequence:

(i) The error of the WCS algorithm is less than that of the CS one;

(ii) If compared with the spectral curve produced with the CS algorithm, the curve reconstructed using the WCS algorithm is closer to the original curve, and the relevant fitting quality is higher. The spectral reflectance reconstruction accuracy based on the white-balance correction is greater than that of the method with no correction.

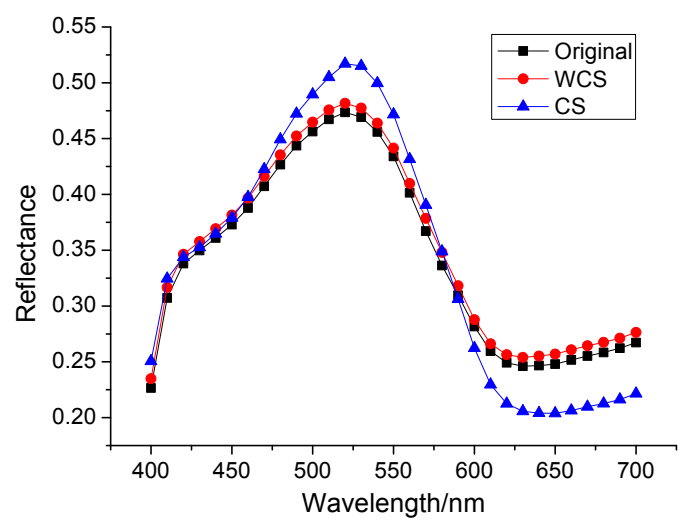

Fig. 4. Comparison of results obtained with different spectral reflectance reconstruction algorithms (see the text).

\subsection{The influence of wavelength interval}

To study the influence of the wavelength interval, we have chosen the Munsell card as a basis for the training sample set. The spectral reflectance range is $400-700 \mathrm{~nm}$, and the sampling intervals are taken to be 5,10 and $20 \mathrm{~nm}$. The spectral reflectance of the training sample is processed at different sampling intervals, using the principal component analysis. As a result, the first three principal components and the singular value of the training sample set have been obtained. The contribution rate of the first three principal components has been calculated, and the spectral reflectance of the surface of the untested sample has been reconstructed using our WCS algorithm. The relationship between the reconstruction erros and the sampling interval is demonstrated in Table 2 .

The following conclusions of our analysis are worthwhile:

(i) The cumulative contribution rates of the first three principal components are 98.55, 98.54 and $98.53 \%$ respectively for the wavelength intervals 5,10 and $20 \mathrm{~nm}$, i.e. the rate decreases with increasing interval;

Ukr. J. Phys. Opt. 2016, Volume 17, Issue 3 
(ii) The reconstruction errors for the first three principal components are equal to 0.000388 , 0.000686 and 0.003256 for the sampling intervals 5,10 and $20 \mathrm{~nm}$, i.e. increasing interval increases this error;

(iii) The reconstructed colour difference increases with increasing wavelength interval;

(iv) The fitting quality coefficient decreases with increasing wavelength interval.

Table 2. Relationships between the reconstruction accuracy of our WCS method and the wavelength sampling interval.

\begin{tabular}{|c|c|c|c|}
\hline Interval, nm & $R M S E$ & $\Delta E$ & $G F C$ \\
\hline 5 & 0.000388 & 0.0199 & 0.9929 \\
\hline 10 & 0.000686 & 0.0226 & 0.9705 \\
\hline 20 & 0.003256 & 0.0268 & 0.9537 \\
\hline
\end{tabular}

\subsection{The influence of number of iterations}

For investigating the influence of iteration number on our results, we have selected six-channel response values of the samples. The Munsell colour card has been chosen as a training sample, and the first six principal components of the training samples have been taken to fix the basis function vector. The spectral reflectance for the woman face image has been reconstructed using our WCS algorithm. The relationship between the reconstruction error and the number of iterations is shown in Fig. 5. It is obvious that the reconstruction error decreases with increasing number of iterations. This error can be minimized when the number of iterations becomes close to the sparsity of the spectral reflectance.

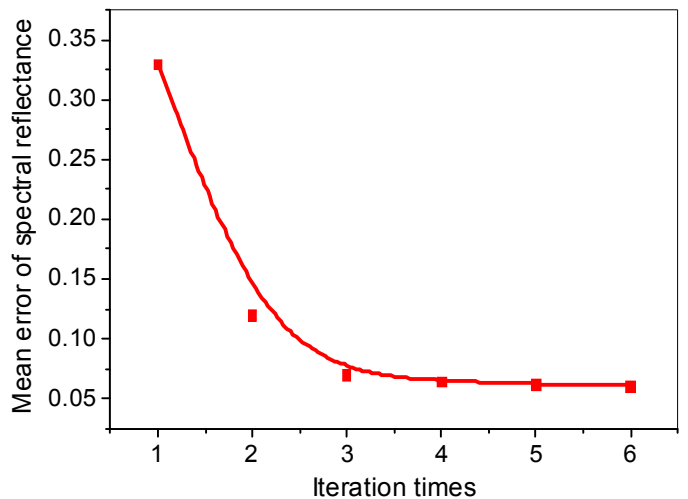

Fig. 5. Dependence of root-mean-square error of our WCS method on the number of iterations (see the text).

\subsection{The effect of number of principal components}

To study the effect of the number of principal components, we have been selected RC24, SG140 and Munsell colour training sample sets. In general, all of those three kinds of the training samples are consistent in their colour space distributions. However, the similarities in the colour spatial distributions of the X-Rite 24-colour card and the 140-colour testing sample SG140 are somewhat smaller, while the Munsell colour space distribution is located in the centre of the radial distribution. It is also more similar to the multispectral testing image. Therefore, the Munsell colour has some advantages in this respect.

Through the principal component analysis, the first six principal components of the multispectral reflectance data of the training samples have been derived, which constitute the basis 
function vector to reconstruct the spectral reflectance. The average transformation matrix $M$ and the basis function vector $B$ have been obtained using respectively the formula $M=L \times D$ and the principal component analysis. The spectral reflectance of the untested sample has been reconstructed with our WCS algorithm (see Eq. (11)). The relationship between the error value and the number of principal components is shown in Fig. 6. The following conclusions are straightforward:

(i) The spectral reconstruction errors are bigger when the process is based on the six principal component of the RC24 colour cards and the 140-colour training samples. The error becomes the lowest in case of the principal components of the Munsell colour card;

(ii) With the same training sample, the error decreases with increasing number of the principal components. When this number increases to three, the spectral reflectance reconstruction error based on our WCS method changes little (see Fig. 6). The reason is that the contribution rate of the first three principal components is more than $95 \%$.

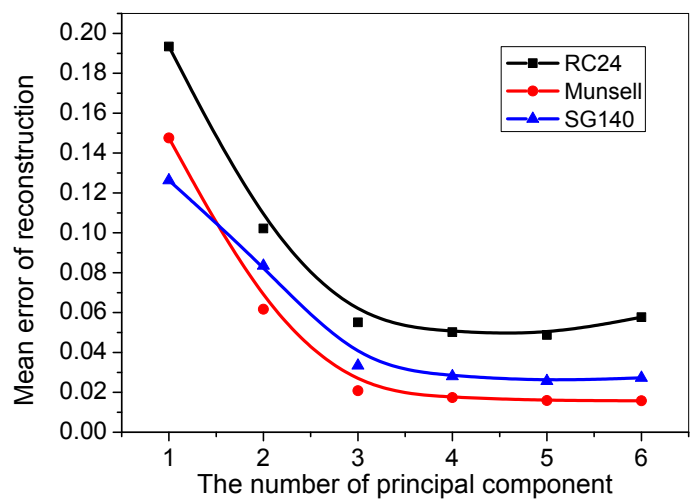

Fig. 6. Dependence of reconstruction error of our WCS method on the number of principal components (see the text).

\section{Conclusions}

In this study we have suggested a mapping function of the well-known compressive sensing algorithm, which is based on the principal component analysis. The average transformation matrix has been obtained by structuring a series of modulated light sources to illuminate the standard whiteboard. This has enabled us to avoid the influence of training samples on the average transformation matrix, improve the reconstruction precision and reduce the amount of necessary computations. As compared to the traditional CS algorithm with no white balance involved, we employ the white-balance processing to regulate indirectly the lighting components in the image and to provide consistency of the RGB images obtained with different illumination sources. Therefore a single mapping of the RGB image onto the spectral reflectance is only needed. As a result, the spectral reflectance can be reconstructed with notably lower root-mean-square error and higher fitting quality. Moreover, our WCS algorithm of spectral reconstruction ensures a better spectrum-matching effect, smaller errors and improved spectral reconstruction performance, if compared with the traditional CS method.

To obtain the vector of the basic functions of the spectral reflectance reconstruction using the principal component analysis, we have used the Munsell colour card as a training sample. Then the dimensionality of high-dimensional spectral reflectance is reduced. We have reconstructed a number of testing samples based on our improved WCS algorithm.

By comparing the root-mean-square error and the colour difference, one can conclude that 
the reconstruction accuracy based on our WCS algorithm is better than that of the CS one. We have also studied some factors which can influence the results of our method. In particular, we have demonstrated how the reconstruction precision of the WCS algorithm is affected by the number of principal component, the wavelength sampling interval and the number of iterations. It is shown that the spectral reconstruction error and the colour difference increase with increasing wavelength interval, so that the reconstruction precision falls down. At the same time, the spectral reconstruction error decreases with increasing number of iterations.

\section{Acknowledgments}

This study is supported by the National Basic Research Program of China (973 Program) under the Grant No.2015CB352004, the National Natural Science Foundation of China (Grant No.61405115, 61378035), the Natural Science Foundation of Shanghai (Grant No. 14ZR1428400), Innovation Project of Shanghai Municipal Education Commission (Grant No. 14YZ099).

\section{References}

1. Day E A, Berns R S and Taplin L A, 2004. A psychophysical experiment evaluating the color accuracy of several multispectral image capture techniques. Imaging Sci. Technol. 48: 93-104.

2. Zhang X D, Wang Q, Yang G F, Wu L, 2013. Key technologies review of the spectral color management system. China Printing and Packing Study. 5: 10-17.

3. Jiang B W, 2012. Method of multi-spectral images reduce-dimensions based on PCA. Inform. Technol. 8: $98-101$.

4. Takase K, Tsumura N, Nakaguchi T and Miyake Y, 2005. Fast estimation algorithm for calculation of reflectance map based on Wiener estimation technique. Opt. Rev. 12: 20-24.

5. Maloney L T and Wandell B A, 1986. Color constancy: a method for recovering surface spectral reflectance. J. Opt. Soc. Amer. A. 3: 29-33.

6. Zhang L H, Liang D, Pan Z L and Ma X H, 2015. Study on the key technology of reconstruction spectral reflectance based on the algorithm of compressive sensing. Opt. Quant. Electron. 47: 1679-1692.

7. Zhang Leihong, Liang Dong, Li Bei, Kang Yi, Pan Zilan, Zhang Dawei and Ma Xiuhua, 2016. The study of key technology on spectral reflectance reconstruction based on the algorithm of adaptive compressive sensing. Laser Phys. 26: 045201.

8. Bioucas-Dias J M and Figueiredo M A T, 2007. A new TwIST: two-step iterative shrinkage/ thresholding for image restoration. IEEE Trans. Image Process. 16: 2992-3004.

9. Romberg J, 2008. Imaging via compressive sampling. IEEE Signal Process. Mag. 25: 14-20.

10. Donoho D L, 2006. Compressed sensing. IEEE Trans. Inform. Theory. 52: 1289-1306.

11. Amirshahi S H and Amirhahi S A, 2010. Adaptive non-negative bases for reconstruction of spectral data from colorimetric information. Opt. Rev. 17: 562-569.

12. Candès $\mathrm{E} \mathrm{J}$, Romberg $\mathrm{J}$ and Tao $\mathrm{T}, 2006$. Robust uncertainty principles: Exact signal reconstruction from highly incomplete frequency information. IEEE Trans. Inform. Theory. 52: 489-509.

13. Nguyen R M H, Prasad D K and Brown M S, 2014. Training based spectral reconstruction from a single RGB image. Lecture Notes in Comp. Sci. 8695: 186-201.

14. Finlayson H S and Trezzi E, 2004. Shades of gray and colour constancy. In: Proceedings of IS\&T/SID Twelfth Color Imaging Conference. p. 37-41.

15. Spectral Image Database. University of Eastern Finland Color Group [EB/OL]. http://www.uef.fi/spectral/spectral image database. 
Zhang Leihong, Liang Dong, Li Bei, Kang Yi, Zhang Dawei and Ma Xiuhua 2016. Spectral reflectance recovery from a white-balanced RGB image based on the algorithm of compressive sensing. Ukr.J.Phys.Opt. 17: $112-123$

Анотація. У роботі вивчаються методи відновлення спектрального відбивання зображень. Для точнішого відтворення спектральної відбивної здатності $і$ більш реалістичного відтворення кольорів ми одержуємо $m$. зв. середню матрицю перетворення, використовуючи низку різних модульованих джерел для освітлення стандартної "білої дошки”. Для усунення впливу иих джерел світла на RGB-зображення ми спочатку використовуємо алгоритм балансу білого, щоб стандартизувати інформацію про сиену, отримуючи у цей спосіб однорідне RGB-зображення для випадку різних джерел. Далі ми формуємо відповідність (мапу) між RGB-зображенням $i$ спектральним відбиванням, виходячи з алгоритму стиснутого зондування та аналізу головних компонент $i$, нарешті, реконструюємо спектральне відбивання тестового зразка. Ми порівнюємо точність реконструкиії зображення для нашого алгоритму стиснутого зондування, заснованого на калібруванні балансу білого, із результатами, отриманими $з$ використанням псевдоінверсного методу i традиційного варіанту алгоритму стисненого зондування. Результати моделювання показують, що за тих же умов реконструкиії залишкові похибки i відмінності в кольорах для нашого поліпшеного алгоритму менші, ніж відповідні величини для інших двох алгоритмів. Тому запропонований нами алгоритм відновлення переважає иі методи і може забезпечити вищу продуктивність відтворення кольорів зображень. 